\title{
Development of Algorithm for Preservation of Heritage Murals and Painting Using Image Processing
}

\author{
S. Lalithakumari, Pandian R. \\ Department of Electrical and Instrumentation Engineering, Sathyabama University, India
}

\begin{tabular}{l} 
Article Info \\
\hline Article history: \\
Received May 8, 2018 \\
Revised Jul 19, 2018 \\
Accepted Aug 2, 2018 \\
\hline
\end{tabular}

Keywords:

Culture Heritage

Image Archiving

Murals

Presevation

\begin{abstract}
Image archiving and preservation finds extensive application in culture heritage murals. The study of cultural heritage is of the extreme importance at national and international levels. Not only global organizations like UNESCO but also museums, libraries, culture, temples and private initiatives are working in these directions. During the last three decades, researchers in the field of imaging discipline have started to contribute an increasing set of algorithms for cultural heritage; in that way providing indispensable support to these efforts. A better comparison of the different compression methods presented in this proposed work for culture Heritage mural images. Compression methods usually applied some method to reduce the number of components within each spectrum. The effectiveness of mural image archiving and preservation is analyzed based on 2-D wavelets filtering. The optimum algorithm is also found based on the results.
\end{abstract}

Copyright (0) 2018 Institute of Advanced Engineering and Science. All rights reserved.

\section{Corresponding Author:}

S. Lalithakumari,

Department of Electrical and Instrumentation Engineering,

Sathyabama University,

Chennai,Tamil Nadu, India

Email: rpandianme@rediffmail.com

\section{INTRODUCTION}

The signal, image, and video processing, computer vision, 3D modeling, and graphics technologies are nowadays broadly employed to capture, analyze, conserve, virtually or physically restore, document, classify, recognize, and render cultural artifacts. These contain historic buildings and monuments, archaeological sites and finds, works of art (paintings, frescoes, sculptures, decorative items, etc.), manuscripts, music score manuscripts, photographs or photographic negatives, films, and other entities of artistic, historical, or archaeological importance. In this research can be grouped into two main strands. On the one hand, tools aim to provide easy access to cultural heritage by both the general public and scholars. On the other hand, a substantial body of work wants to ensure its preservation for future generations. An important issue that expedited andboosted theutilization ofimageandvideo proces techniques in cultural heritage applications were the initiation of in depth conversion campaigns by public establishments, museums, libraries, and archives throughout the last years. As a result, an enormous quantity ofculturerelated info is currently digitally keep,and therefore amenabletodigital Process [1-4].

The field of image and video processing for cultural heritage encompasses a large variety of topics, such as high-resolution 2D and 3D digital capture and rendering of artworks, digital restoration, enhancement, recognition, and classification of features, structures and content in cultural heritage visual data, creation of large-scale multimedia databases of artworks, and user-centered heritage-related visual or multimedia applications. Indeed, image and video processing techniques can significantly improve and make more efficient many aspects of traditional preservation, archival, study, and fruition of our cultural heritage and, very interestingly, can also provide answers to emerging needs [5-8]. Moreover, they have made feasible the creation of new applications and tools, which would otherwise be impossible to realize. The paper is structured as 
follows. Section 2 describes the Transform technique and. Results and discussion are explained in Section 3 and this research work is concluded in Section 4.

\section{WAVELET TRANSFORM}

In wavelet analysis, pictures are archived by a set of basic functions. A single prototype function defined the mother wavelet is used for deriving the basis function, by translating and dilating the mother wavelet. The wavelet transform can be termed as a decomposition of an image in the time scale plane. In this work, Daubechies, symlets and coifles are proposed. The basic and compact wavelet, which is projected by Daubechies is an orthonormal wavelets, which is defined as Daubechies wavelet. It is considered with extremely phase and the highest number of vanishing moments for a given support width. Associated scaling filters are minimum-phase filter. Daubechies wavelets are normally used for solving fractal problems, signal discontinuities, etc. The symlets are almost symmetrical wavelet, which is projected by Daubechies as modifications to the $\mathrm{db}$ family. Daubechies proposed modifications of her wavelets that raise their symmetry can be increased while retaining great simplicity. The symlets have properties similar to daubechies [9][10].

\section{RESULTS AND ANALYSIS}

In this work, the mural image is used for the proposed algorithms. The Discrete wavelet transform based decomposition was performed on the image. Symlet2, coiflet 2 and daubecheis 2 are implemented for decomposing the mural image. Levels1, 2, 3 and 4 of decomposition are adopted. After the decomposition, Zero tree Encoding is employed. The capability of the compression algorithms is evaluated in terms of MSE, PSNR, compression ratio and Bits per pixel. This is tabulated in Table 1. The Quality of the reconstructed image is measured in terms of mean square error (MSE) and peak signal to noise ratio (PSNR) ratio. The effectiveness of the compression can be analyzed using the compression ratio and bits per pixel. The MSE is often called reconstruction error variance $\square \mathrm{q} 2$. The MSE between the original image and the reconstructed image $g$ at the decoder is defined as:[10].

$$
\mathrm{MSE}=\sigma^{2}=1 / \mathrm{N}^{*} \text { summation of }(\mathrm{f}[\mathrm{j}, \mathrm{k}]-\mathrm{g}[\mathrm{j}, \mathrm{k}])^{2}
$$

where the sum over $\mathrm{j}$, $\mathrm{k}$ denotes the sum over all pixels in the image and $\mathrm{N}$ is the number of pixels in each image. From that the peak signal-to-noise ratio is defined as the ratio between signal variance and reconstruction error variance. The PSNR is measured in terms of decibels $(\mathrm{dBs})$ is given

$\mathrm{PSNR}=10 \log _{10}\left(255^{2} / \mathrm{MSE}\right)$

Generally when PSNR is $40 \mathrm{~dB}$ or greater, then the archived and the compressed murals are virtually indistinguishable by human eyes. The compression ratio of the image is given by No. of bits in original image/ No. of bits in compressed image. The original murals, compressed murals with Symlet2, level 1 decomposition and level 4 decomposition are shown in Figure 1 and Figure 2 respectively.

Table 1. Performance Evaluation for Decomposition Levels

\begin{tabular}{cccc}
\multicolumn{4}{c}{ Decomposition Level 1 } \\
\hline Transforms & DB 2 & SYM 2 & COIF 2 \\
\hline PSNR (dB) & 52.1 & 59.68 & 58.47 \\
BPP & 20.23 & 21.99 & 22.47 \\
CR $(\%)$ & 84.3 & 94.79 & 93.64 \\
MSE & 0.51 & 0.088 & 0.09 \\
\hline
\end{tabular}

\begin{tabular}{cccc}
\multicolumn{4}{c}{ Decomposition Level 2 } \\
\hline Transforms & DB 2 & SYM 2 & COIF 2 \\
\hline PSNR (dB) & 50.55 & 50.55 & 50.41 \\
BPP & 16.93 & 16.93 & 16.29 \\
CR $(\%)$ & 70.65 & 70.65 & 67.89 \\
MSE & 0.573 & 0.573 & 0.6 \\
\hline
\end{tabular}

\begin{tabular}{cccc}
\multicolumn{4}{c}{ Decomposition Level 3 } \\
\hline Transforms & DB 2 & SYM 2 & COIF 2 \\
\hline PSNR (dB) & 44.83 & 44.83 & 44.84 \\
BPP & 10.68 & 10.68 & 10.04 \\
CR(\%) & 44.49 & 44.49 & 41.84 \\
MSE & 2.14 & 2.14 & 2.14 \\
\hline
\end{tabular}

\begin{tabular}{cccc}
\multicolumn{4}{c}{ Decomposition Level 4 } \\
\hline Transforms & DB 2 & SYM 2 & COIF 2 \\
\hline PSNR (dB) & 43.82 & 44.82 & 44.8 \\
BPP & 10.47 & 10.49 & 10.35 \\
CR $(\%)$ & 43.61 & 43.64 & 43.13 \\
MSE & 2.2 & 2.14 & 2.15 \\
\hline
\end{tabular}




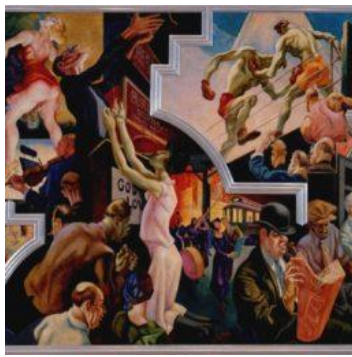

Figure 1.Original image

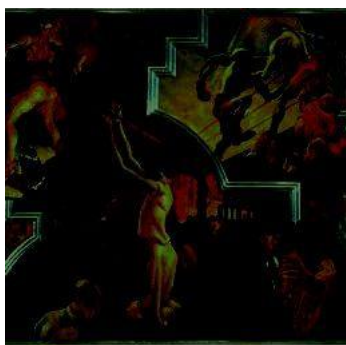

Figure 2. Image archived at decomposition level 4

The PSNR values are approximately same and very low, compared to levels $1 \& 2$. The bits per pixel values are also created and the variations in its values are also following the PSNR value, since, it is related to the PSNR. The mean square error is also raised if the level of decomposition is increased. It is wise to obtain a least value of error, which is also given by symlet1 with the first level of decomposition

\section{CONCLUSION}

The main objective of this proposed work is to archive and compress the mural images with high quality, which is obtained by the transformation of symlet wavelet with the encoding of EZW. It is also achieved with the first level of decomposition. The bits per pixel of the different proposed algorithms are also decreased if the level of decomposition is increased. The performances are varied, mainly due to the nature of the mother wavelets, used in this work. The Levels of decomposition also plays an important role in the storage of murals, which is proven by the values of compression and PSNR values.

\section{REFERENCES}

[1] Pandian, R \& T. Vigneswaren. "Adaptive wavelet packet basis selection for zerotree image coding." International Journal of Signal and Imaging Systems Engineering 9 (2016): 388-392.

[2] Pandian, R. "Evaluation of image compressionalgorithms." 2015 IEEE Underwater Technology (UT) Chennai (2015),1-3.

[3] Pandian $\mathrm{R}$,Vigneswaran $\mathrm{T}$ and Lalitha Kumari $\mathrm{S}$ Characterization of $\mathrm{CT}$ Cancer Lung Image Using Image Compression Algorithms and Feature Extraction, J SCI IND RES, Vol. 75 (2016),747-751.

[4] Rogerl Claypoole et al, "Non linear Wavelet transform for image coding via lifting." IEEE, 2003.

[5] A.R.cadder bank, "Loss less image compression using integer to integer wavelet transforms." IEEE 1997.

[6] Minh N. Do, "Countourlet transform: An efficient directional multi resolutional image representation", 2005.

[7] Gemma paella, "Adaptive lifting schemes combiming semi norms for lossless image compression." IEEE, 2005.

[8] Chenwei Deng, Weisi Lin, Jianfei Cai. Content-Based Image Compression for Arbitrary-Resolution Display Devices. IEEE Transactions on Multimedia 14(4): 1127-1139 (2012).

[9] MA Hong-wei, WANG Bin, Application of Wavelet Transform to Signal de-noising in Ultrasonic Testing, NDT, Volume 26 Number 2, Feb 2004, P68-71

[10] Zhou Wei, Advanced Technology of Wavelet Analysis Based on MATLAB, Published by XiDian University Press,Xi'an, 2006

\section{BIOGRAPHIES OF AUTHORS}
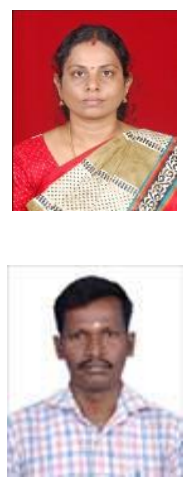

S.Lalitha Kumari has completed graduation from Bharathidasan University, Thiruchirappalli (1993) in Electronics and Communication Engineering Discipline. She obtained her post Graduation in Power Electronics from Bharathidasan University, Thiruchirappalli (1999). Presently she is working as an Asst.professor in the Department of EIE of Sathyabama University, Chennai. She is also pursuing her Doctorate at Sathyabama University, Chennai.. Her research interest includes Signal Processing and Soft Computing.

R.Pandian has completed graduation from Madra University, Chennai (1999) in Electrical and Electronics Engineering Discipline.he obtained her post Graduation in Applied Electronics from Anna University, Chennai (2007). Presently she is working as an Asst.professor in the Department of EIE of Sathyabama University, Chennai. He is also pursuing her Doctorate at Sathyabama University, Chennai.His research interest includes Image Processing. 